Ein im großen aus denselben Puppen dargestelltes Rohöl war dunkelbraun und besab einen entfernt an Fischöl erinnernden Geruch. Durch Filtration über Bleicherde wurde eine bedeutende Aufhellung des Öles erzielt. Beim Stehen schieden sich ebenfalls feste Bestandteile aus; diese waren jedoch amorph und stellten eine voluminöse flockige Masse dar. Dies mag zum Teil dadurch verursacht sein, daß dem Handelsprodukte noch Spuren des Extraktionsmittels anhafteten.

Das im großen dargestellte und gebleichte Öl gab die folgenden Zahlen:

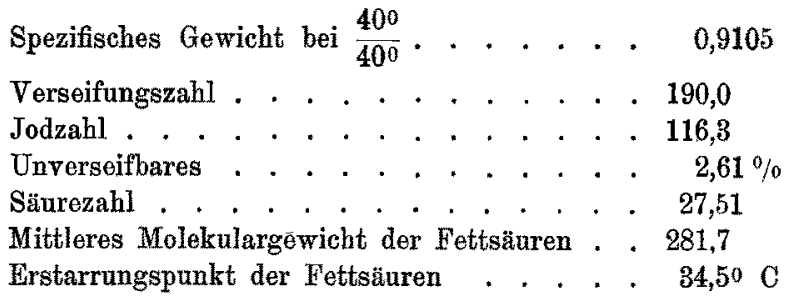

Die bedeutend höhere Säurezahl des ersteren Öles ist natürlich der weiter fortgeschrittenen Zersetzung der von mir extrahierten Puppen zuzuschreiben ${ }^{1}$ ).

Da beträchtliche Mengen dieses Öles leicht erhältlich sind, so dürfte einer Verwendung desselben in der Seifenindustrie für Seifen geringer Qualität nichts im Wege stehen.

1) Vergl. J. Lewkowitsch, Chemische Technologie und Analyse der Öle, Fette und Wachse. Braunschweig 1905, Bd. I, S. 27.

\title{
Zu der Veröffentlichung von A. Beythien betr. Garantol. Von
}

E. Utescher-Hamburg.

Im Heft 8 dieser Zeitschrift vom 15. Oktober 1906 veröffentlicht A. Beythien eine Mitteilung aber Garantol, in welcher auch mein Name genannt wird.

$\mathrm{Zu}$ dem Inhalt habe ich Folgendes festzustellen:

1. Ich stehe mit der Garantol-Gesellschaft m. b. H., welche von Herrn A. Beythien als eine sogenannte Garantol-Gesellschaft m. b. H. bezeichnet wird, in keinerlei Beziehung, im Gegensatz zu einer Gesellschaft gleichen Namens, welche vor längerer Zeit anf meine Veranlassung aufgelöst ist. Ich habe insbesondere auch keinen Einfluf auf die Fabrikationsweise des Garantols; ich habe der Garantol-Gesellsehaft aukerdem wiederholt untersagt, daßs sie sich irgendwie auf mich bei ihrer Fabrikation oder überhaupt in bezug auf ihr Gesehaftsunternehmen bezieht.

2. Das Patent No. 75671 ist nicht, wie die übrigen von A. Beythien genannten Patente, mir erteilt, sondern an August Utescher; die übrigen Patente sind mir erteilt.

3. Die Frage, ob durch das Patent No. 119574 alle Vorteile der ursprünglichen Erfindung aufgegeben sind, das kann nur der beurteilen, der hierüber Versuche gemacht hat; nach der Mitteilung von A. Beythien muk ich annehmen, daß er über diese lediglich nach Versuchen zu beurteilende Frage ohne jeden $\nabla$ ersuch geurteilt hat.

4. Fast sämtliche Benutzer des Patentes 119574, welchen das Recht der Benutzung dieses Verfahrens von mir gegeben ist, hatten früher mit Kalk nach der üblichen Methode Eier eingelegt; diese Benutzer sind also Sachverständige, wenm sie beide Verfahren verglichen. 
haben, und das Verfahren 119574 in Übereinstimmung mit meinen Versuchen als zweckmäfsiger anerkennen.

5. Niemals ist den Interessenten gegenüber von mir ein Geheimnis gemacht worden, daf es sich bei dem Verfahren 119574, wie überhaupt bei den sämtlichen hier in Frage kommenden Patenten, um Verfahren zur Eierkonservierung handle, welche als verbesserte Kalkverfahren zu bezeichnen sind.

6. Die chemische Analyse des Kalkpräparates zur Eierkonservierung gibt noch keinen Aufschluf üher den Wert desselben; der Sachverständige weik, daß̉ schon Unterschiede im Löschen des Kalkes erbebliche Unterseliede in der Brauchbarkeit zu Zwecken der Eierkonserviernng bedingen oder doch bedingen können, und zwar dann, wenn nicht in klaren Löswogen eingelegt wird, sondern wenn ein Überschuß zum Decken in verschiedenen Schichten benutzt wird.

7. Es bietet der Technik selbstverständlich keine Schwierigkeiten, die Mischung, welche als "Garantol " in dea Verkehr gebracht wird, so herzustellen, daß dieselbe noch Eisenoxydal enthält. Utbrigens ist auch das Eisenoxyd von Vorteil für das fragliche Verfahren; zunächst bindet es Schwefelwasserstoff, welcher beim Konservieren im größeren Maksstabe regelmäßig in den Einlegebassins auftritt; dann ist es aber leichter suspendierbar, legt sich auf die Eierschalen, wird hierbei förmlich angesangt von der Eierschale, hat also auch eine absehliefende Wirkung. Im übrigen unterrichtet über diese Sache auch die Patentschrift; wer Interesse für die Sache hat, kann sie dort nachlesen.

8. Ich sehe es nicht als meine Aufgabe an, die Garantol-Gesellschaft verteidigen zu wollen gegen die Vorwürfe, die A. Beythien etwas indirekt derselben macht; ich glanbe aber, daß auch in der Beurteilung der geschäftlichen Seite der Sache Herr Beythien über Dinge urteilt, die er nicht zu beurteilen versteht. Ich überlasse der Garantol-Gesellschaft, sich in dieser Beziehung mit Herrn Beythien auseinander zu setzen. Ich will hier ausdrücklich feststellen, dak ich Herrn Bey hih ien keinerlei persönliche Motive unterschiebe; die Freiheit seines Urteils erklärt sich auch leicht auf andere Weise.

Hamburg 5, den 23. Oktober 1906.

\section{Bemerkungen zu vorstehenden Ausfïhrungen}

des Herrn E. Utescher-Hamburg.

Von

\section{A. Beythien-Dresden.}

Zu 1.: Diese sog. Berichtigung ist keine Berichtigung, denn in meinem Aufsatze ist nirgends davon die Rede, dak Herr Utes $\mathrm{ch}$ er mit der Garantol.Gesellschaft in Beziehung steht. Tatsächlich beruft die genannte Gesellschaft sich darauf, daß sie Patente des Herrn Utescher erworben habe.

Zu 2.: Ob das Patent 75671 Herrn E. Ute scher oder seinem Bruder A giust U t escher erteilt worden ist, erscheint für die Beurteilung des Mittels belanglos.

Zu 3.: Über die Wirksamkeit des Garantols vermag jeder zu urteilen, der die Ergebnisse der chemischen Analyse zu deuten versteht. Praktischer Versuche bedarf es hierzu nicht.

Zu 4.: Die praktischen Sachverständigen, denen Herr Utescher die Entscheidung zuweisen möchte, sind erfahrungsgemüfs sehr leicht geneigt, in Präparaten unbekannter Zusammensetzung geheimnisvolle Stoffe zu vermuten, und kommen daher infolge einer Autosuggestion oft dazu, denselben eine besondere Wirksamkeit gegenüber altbekannten Mitteln zuzuschreiben. Jeder Fabrikant technischer und medizinischer Geheimmittel vermag seinen Erzeugnissen eine Anzahl empfehlender Gutachten beizulegen.

Zu 5.: Es ist nirgends behauptet worden, dab Herr Utescher selbst aus seiner Erfindung ein Geheimnis macht. Ganz im Gegenteil habe ich die Nummern der erteilten Patente ausdrücklich mitgeteilt. 